\title{
INVESTIGATING THE USE OF COASTAL BLUE IMAGERY FOR BATHYMETRIC MAPPING OF INLAND WATER BODIES
}

\author{
G. Mandlburger ${ }^{1,2}$, J. Kremer $^{3}$, F. Steinbacher ${ }^{4}$, R. Baran ${ }^{4}$ \\ ${ }^{1}$ University of Stuttgart, Institute for Photogrammetry, Germany - gottfried.mandlburger@ifp.uni-stuttgart.de \\ ${ }^{2}$ TU Wien, Department of Geodesy and Geoinformation, Austria - gottfried.mandlburger@geo.tuwien.ac.at \\ ${ }^{3}$ IGI mbH, Kreuztal, Germany - j.kremer@igi-systems.com \\ ${ }^{4}$ AirborneHydroMapping GmbH, Innsbruck, Austria (f.steinbacher, r.baran)@ahm.co.at
}

Commission I, WG I/2

KEY WORDS: Multi-media photogrammetry, Laser Bathymetry, Multispectral depth estimation, Shallow Water Mapping

\begin{abstract}
:
In this contribution, we report on an experimental airborne data acquisition with two medium format cameras (Coastal Blue, RGB) and a topo-bathymetric laser scanner for capturing the bathymetry of a dozen of groundwater supplied lakes located near Augsburg, Germany. The specific research question was to investigate whether the use of high-resolution Coastal Blue imagery $(\lambda=400-460 \mathrm{~nm})$ provides added value for mapping bathymetry and characterization of water bottom features. While data processing is still in progress, preliminary results indicate that the blue $(\lambda=420-500 \mathrm{~nm})$ and green $(\lambda=490-570 \mathrm{~nm})$ color channels of the RGB camera are better suited for estimating bathymetry, but the Coastal Blue channel adds an additional water penetrating band increasing the number of useful band combinations with a positive effect on the water bottom classification capabilities. Whereas Coastal Blue channels are rather used from satellite platforms (Landsat 8, WorldView-2) with spatial resolutions in the meter range, our experiment aims at using higher resolution Coastal Blue imagery with a ground sampling distance of around $5 \mathrm{~cm}$ enabling not only spectrally based shallow water depth mapping but also the application of multi-media photogrammetry in high spatial resolution. To the best of our knowledge the use of high-resolution Coastal Blue captured from airborne platforms is novel in the context of mapping shallow water bathymetry.
\end{abstract}

\section{INTRODUCTION}

Today, airborne topo-bathymetric LiDAR (Light Detection And Ranging) is the state-of-the-art for mapping shallow coastal and inland water bodies, provided the water is clear enough to allow the application of optical remote sensing techniques. Water depths are estimated by measuring the round trip time of a short laser pulse within the water column. A green laser $(\lambda=532 \mathrm{~nm})$ is used, as signal attenuation is comparably low at this wavelength (Guenther et al., 2000), while absorption is much higher for longer wavelengths (red, near infrared) which are consequently inappropriate for measuring bathymetry. While shorter wavelengths in the blue and violet domain of the electromagnetic spectrum are comparably well suited for shallow water mapping due to their ability to penetrate the water column (Anderson and Marchisio, 2012), no long-lasting and sufficiently powerful laser sources are available at these wavelengths. However, these wavelengths can well be captured with passive imagery.

Whereas blue $(\lambda=450-500 \mathrm{~nm})$ and green $(\lambda=490-570 \mathrm{~nm})$ are standard bands of an RGB image, modern multispectral satellite sensors like Landsat 8 (Operational Land Imager, OLI) or WorldView-2 provide a so-called Coastal Blue band (WorldView-2: $\lambda=396-458 \mathrm{~nm}$ ). On the one hand, signal attenuation in clear water is theoretically least for this wavelength which makes it most suitable for mapping shallow clear water bathymetry (Miecznik and Grabowska, 2012). On the other hand, it is absorbed by chlorophyll in healthy plants and, therefore, aids in conducting vegetative analysis but it is also influenced by atmospheric scattering (Anderson and Marchisio, 2012). The spatial resolution of the mentioned multispectral satellite bands,

\footnotetext{
${ }^{*}$ Corresponding author
}

however, is moderate (Landsat 8: $30 \mathrm{~m}$, WorldView-2: $1.8 \mathrm{~m}$ ) and is outperformed by modern topo-bathymetric LiDAR sensors with typical point densities in the range of 20 points $/ \mathrm{m}^{2}$ and laser footprint diameters of approximately $40-60 \mathrm{~cm}$ when flown from nominal altitudes of 400-600 m.

The question therefore arises, if high-resolution images from Coastal Blue- and RGB-cameras mounted on an airborne platform together with a topo-bathymetric laser scanner would improve bathymetric mapping in terms of accuracy, reliability, and water bottom classification capabilities compared to using a bathymetric LiDAR sensor only. For this purpose an experiment was planned and an airborne data acquisition was carried out in April 2018 near Augsburg, Germany. In the following, first, the sensor configuration is introduced (Section 2), followed by a description of the data acquisition and the applied data processing steps (Section 3) and, finally, preliminary results are presented and discussed (Section 4). The manuscript is wrapped up with concluding remarks and an outlook on future work on subject matters (Section 5).

\section{SENSOR CONFIGURATION}

The employed hybrid sensor system consisted of two IGI DigiCAM 100 cameras, both of which based on PhaseOne iXURS 1000 cameras (pixel size: $4.6 \times 4.6 \mu \mathrm{m}^{2}$, image size: 11608 $\mathrm{x} 8708$ pixel) and a RIEGL VQ-880-G topo-bathymetric laser scanner (pulse repetition rate: $550 \mathrm{kHz}$, laser beam divergence: $1.1 \mathrm{mrad}$ ). Both DigiCAM cameras were equipped with a Rodenstock $50 \mathrm{~mm}$ wide angle lens (FOV: $56.2^{\circ} / 43.7^{\circ}$ ).

Besides the off-the-shelf RGB sensor featuring a Bayer filter, the focus was laid on the Coastal Blue wavelength. For this purpose, 


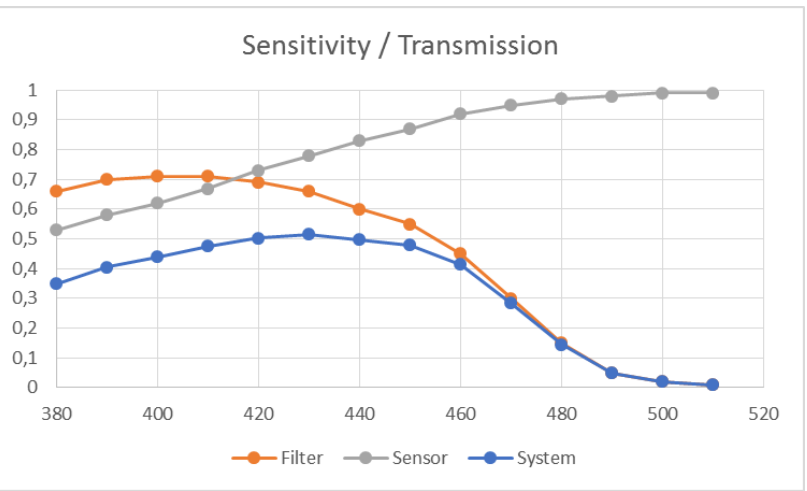

Figure 1. DigiCAM 100 Coastal Blue: Sensitivity/transmission curves of CMOS sensor (gray), filter (orange), and resulting system performance (blue) for different wavelengths [nm]

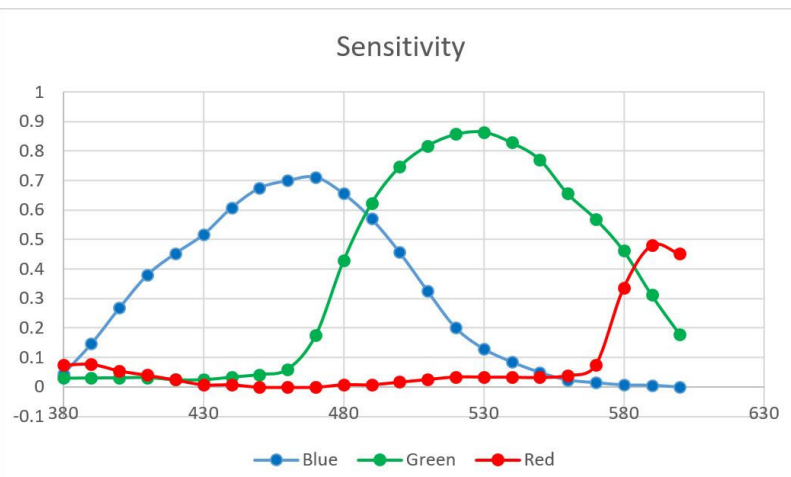

Figure 2. DigiCAM 100 RGB: System performance for red, green, and blue channel over different wavelengths [nm]

a second camera with a pan-chromatic CMOS active-pixel sensor was used and all but the Coastal Blue radiation was blocked by a respective filter mounted in front of the lens. The characteristics of the sensor, the filter, and the resulting system performance are plotted in Figure 1. It can be seen that the peak performance is at $\lambda=430 \mathrm{~nm}$ and the sensitivity drops sharply for wavelengths larger than $460 \mathrm{~nm}$.

For the RGB camera, Figure 2 shows the total system performance of the three bands. The green band, for example has its peak performance exactly at the laser wavelength of $\lambda=532 \mathrm{~nm}$. But while the laser intensity (captured in full waveform profiles) represents the amount of backscattered energy in an extremely narrow spectral band due to the monochromatic property of the laser light, the green channel of the DigiCAM 100 captures radiation within a wider spectral range between $480-590 \mathrm{~nm}$, and hereby the spectral range partially overlaps with the adjacent blue $(410-500 \mathrm{~nm})$ band. Figure 3 shows the integration of the cameras in the airplane.

\section{DATA ACQUISITION AND DATA PROCESSING}

This section details the experimental flight campaign and summarizes the main data processing steps.

\subsection{Data acquisition}

To investigate the feasibility of using high-resolution Coastal Blue imagery for bathymetric mapping, an airborne data acquisition with the sensor configuration described above was carried

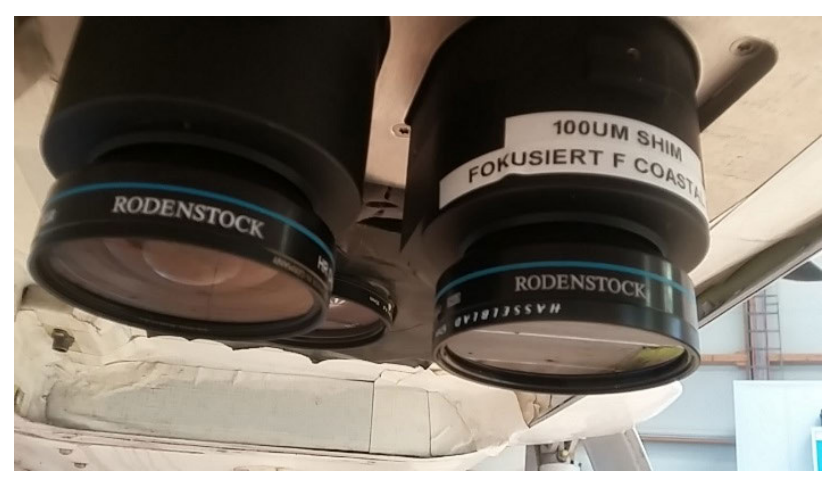

Figure 3. DigiCAM 100 camera heads mounted at the belly of the aircraft (left: RGB, right: Coastal Blue with filter in front of the lens)

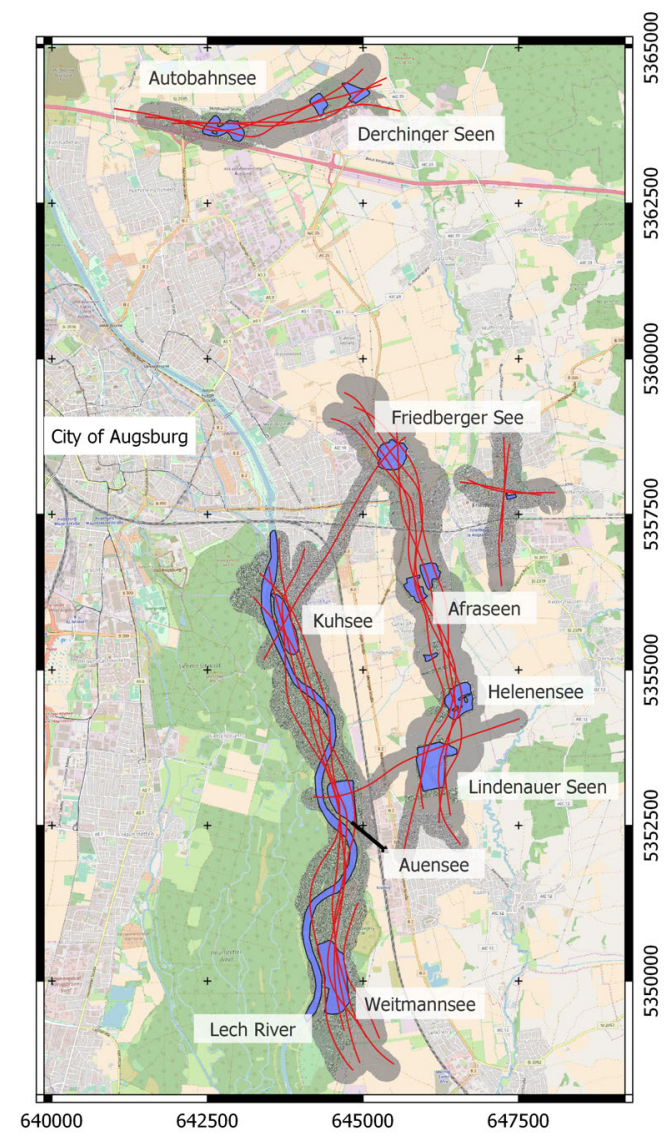

Figure 4. Study area Augsburg; OpenStreetMap superimposed with shaded LiDAR DSM relief map, captured water lakes (blue polygons), and flight trajectories (red); coordinate frame: ETRS89/UTM32

out near Augsburg, Germany, on April, 9th, 2018. A dozen of groundwater supplied lakes and a section of the Lech River were captured in multiple overpasses (cf. Figure 4). The water bodies were chosen due to their variation in water turbidity, ranging from very clear to impenetrably turbid, and bottom reflectivity (bright gravel, dark under water vegetation). The flying height was 500-630 m above ground level (AGL) at a flying speed of about 100 knots $(50 \mathrm{~m} / \mathrm{s})$ yielding a laser footprint diameter on the ground of $55-70 \mathrm{~cm}$ and an average laser pulse density of approximately 20 points $/ \mathrm{m}^{2}$ per strip. The corresponding ground sampling distance (GSD) of the RGB/Coastal Blue images amounts 
to $4.5-5.3 \mathrm{~cm}$. Each image covers an area of $615 \times 460 \mathrm{~m}^{2}$ on the ground for a flying altitude of $630 \mathrm{~m}$ AGL and of $520 \times 390 \mathrm{~m}^{2}$ for the flight lines captured from $500 \mathrm{~m}$ AGL, respectively. As the width of the individual water bodies is in general $\leq 400 \mathrm{~m}$, the images contain dry land areas at both sides of the lakes for all centered flight lines, thus providing enough reliable areas for proper image orientation not effected by image ray refraction at the air-water interface.

In total, approximately 20 flight strips and more than 6000 images with an overlap of $90 \%$ have been recorded in two flight sessions. With the chosen frame rate of $0.7 \mathrm{~s}$ the mean distance between consecutive exposures was ca. $35 \mathrm{~m}$ resulting in a mean stereo pair ray intersection angle of $3-5^{\circ}$ depending on terrain undulations and slightly varying flight velocities. As narrow intersection angles have a negative impact on the photogrammetric height accuracy, stereo pairs were also selected leaving one image out, thus, simulating a standard $80 \%$ forward overlap. Each water body was captured in at least four flight strips (cf. Figure 4) providing highly redundant data.

The achieved LiDAR penetration depth was between 1.5-7.5 m depending on water turbidity and bottom reflectance. Table 1 summarizes the properties of selected lakes. The turbidity estimate is a subjective measure between 0-100 derived from aerial photo interpretation. Whenever no full bottom coverage was achieved, the $>$ sign in column depth denotes the maximum laser penetration depth.

In addition to the airborne data 12 control points (black and white checker board targets) were measured at Friedberger See and Autobahnsee with a Trimble RTK GNSS (Global Navigation Satellite System) receiver. In addition, radiometric control patches (light gray/black stage curtains with calibrated reflectance, size: 3 $\mathrm{x} 4 \mathrm{~m}^{2}$ ) were laid out for radiometric correction of the imagery in post processing. The checker board targets were used as control points for the image bundle block adjustment (cf. Section 3.2).

\begin{tabular}{lccccccr}
\hline water body & turbidity & \multicolumn{7}{c}{ gravel } & \multicolumn{7}{c}{ bright } & \multicolumn{3}{c}{ dark length width depth } \\
& {$[\%]$} & {$[\%]$} & {$[\%]$} & {$[\%]$} & {$[\mathrm{m}]$} & {$[\mathrm{m}]$} & {$[\mathrm{m}]$} \\
\hline Autobahnsee & 10 & 30 & 10 & 60 & 650 & 350 & 4.6 \\
Derchinger Seen & 90 & n.a. & n.a. & n.a. & 400 & 350 & $>2.0$ \\
Friedberger See & 10 & 20 & 50 & 30 & 550 & 450 & $>7.5$ \\
Afraseen & 10 & 25 & 60 & 15 & 320 & 230 & 4.9 \\
Kuhsee & 10 & 50 & 50 & 10 & 1000 & 230 & 4.5 \\
Auensee & 20 & 10 & 20 & 70 & 650 & 380 & 6.8 \\
Helenensee & 10 & 25 & 60 & 15 & 500 & 420 & 7.0 \\
Weitmannsee & 10 & 25 & 50 & 25 & 1120 & 370 & 6.8 \\
\hline
\end{tabular}

Table 1. Dimension and properties of selected lakes

\subsection{Data processing}

Processing of the topo-bathymetric LiDAR data involved the following steps:

- Processing of the raw sensor measurements in the manufacturer's software RiProcess including multiple-time-around resolution of the laser echoes, filtering of after pulses, direct georeferencing based on the trajectory and the initial boresight alignment, and export of the point cloud in LAS format.

- Rigorous strip adjustment including time dependent trajectory correction (Glira et al., 2016) and checking of the residual strip height differences (Ressl et al., 2008) using the scientific laser scanning software OPALS (Pfeifer et al., 2014).
- Filtering of isolated points stemming from sporadic reflections at particles in the atmosphere and remaining after pulses.

- Semi-automatic derivation of water surface height models in $10 \mathrm{~m}$ grid spacing for each lake individually based on manually defined initial heights and the statistic approach of Mandlburger et al. (2013) followed by refraction and runtime correction of the submerged laser echoes according to Snells' law.

- Derivation of a Digital Terrain Model (DTM) of both the bare land surface and the submerged topography based hierarchical robust interpolation (Kraus and Pfeifer, 1998).

The image data processing pipeline consisted of:

- Camera calibration and bundle block adjustment based on structure from motion (automatic extraction of image tie points, tie point matching, estimation of interior and exterior orientation, absolute georeferencing via manual control point measurement) using the Pix4D mapper software. For each lake, individual blocks have been computed for RGB and Coastal Blue to study the impact of the radiometry or texture, respectively, on the geometric quality of the image orientation and the point clouds derived thereof.

- Through-water dense image matching based on stereo images using the SURE software (Rothermel et al., 2012; Wenzel et al., 2013).

- Post-processing of the submerged points including refraction correction and smoothing (Mandlburger, 2018). Again, these steps were performed independently for the RGB and Coastal Blue images.

- Error assessment of the Dense Image Matching results compared to the LiDAR DTM as reference. This in-depth assessment, however, is currently work in progress.

In addition to this geometry oriented processing task, a radiometric analysis was carried out for selected RGB/Coastal Blue images. Next to the individual bands (coastal, blue, green, red) different features derived from combinations of the raw bands or the (natural) logarithms of the bands were tested. The logarithm was hereby used as signal attenuation within the water column follows an exponential law (Lyzenga et al., 2006). While the feature images themselves allow a visual interpretation, the textural diversity of the individual fearures was assessed quantitatively by calculating the mean, variance, and Shannon entropy (Shannon, 2001) for all image pixels within kernels of $7 \times 7$ pixels. To study the different signatures over land and water, the dry and submerged image areas were separated based on manually digitized water masks.

\section{PRELIMINARY RESULTS AND DISCUSSIONS}

While data processing is still ongoing, first preliminary results have already been obtained and are presented in the following.

Figure 5 shows a vertical section of the LiDAR point cloud at the western waterside of Autobahnsee. The displayed points stemming from two different flight strips are colored by classification (blue/green: above/below waterline, brown: isolated points) and represent an intermediate state of data processing after strip co-registration (strip adjustment), isolated point detection, water level estimation, but before refraction correction and DTM filtering. The fact that only a single layer of points is visible in Figure 5 is an indication for a precise fit of the individual flight 


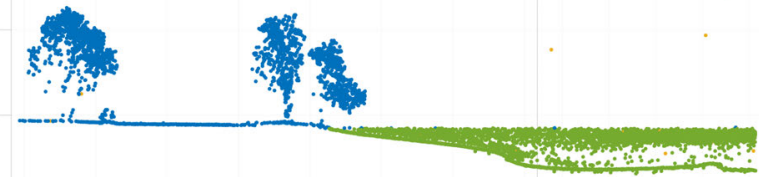

Figure 5. Section view of LiDAR point cloud of Autobahnsee (western shoreline). Points colored by intermediate classification into: point from above (blue) and below (green) the water surface and isolated points (brown)

strips. The residual height error between the overlapping flight strips after strip adjustment, measured as DEM (Digital Elevation Model) height differences at smooth bare land areas amount to $0.0 \pm 0.06 \mathrm{~m}$ (mean \pm std.dev.) for the Autobahnsee sub-block. The LiDAR blocks of all other lakes resulted in similar deviations (no bias, std.dev. $<10 \mathrm{~cm}$ ). Due to high flight dynamics, time dependent trajectory correction (Glira et al., 2016) was necessary to obtain a satisfactory strip fitting precision. It is noted here that, to date, only a relative strip-to-strip alignment was carried out. Absolute block orientation is work in progress based on the image bundle block, which itself is georeferenced via the GNSS control points.

While most of the clutter points from reflections in the atmosphere could successfully be detected with a simple volumetric point density based approach (cf. brown points in Figure 5), still sporadic points just below the actual surface remained in the dataset. These points are referred to as after pulses as they stem from a secondary peak in the system waveform (i.e. the shape of the outgoing pulse). While most of these points have already been removed during initial data processing in the software of the scanner manufacturer (RiProcess), detection of the remaining after pulses was necessary before DTM filtering as these belowsurface points would have had a high influence on the run of the DTM surface. The below-surface points could be successfully identified by estimating the local point density in a cylindrical neighborhood (radius: $2 \mathrm{~m}$, cylinder height: $\pm 5 \mathrm{~cm}$ ) for each point.

After these preparations the remaining LiDAR related processing steps (water surface model estimation, refraction correction, DTM filtering and interpolation, water depth calculations) did not pose further problems and followed standard processing chains. Figure 6 shows the final $50 \mathrm{~cm}$ DTM of both the bare land surface and the submerged topography as a color elevation map (top). In addition, the height precision (i.e. standard deviation of the grid heights interpolated from all points classified as ground with a moving least squares interpolation) is plotted in the bottom part of Figure 6. Within the water area, the brighter (yellow) color tones indicate lower height precision corresponding to areas with submerged vegetation. Lake Autobahnsee features various types of under-water vegetation with different plant density. Whereas in some areas, the vegetation is too dense for canopy penetration, in other areas multiple laser returns from the top of the vegetation and the ground below are obtained. This can clearly be seen in the underlying shaded relief map of Figure 6. It is noted here that the short pulse length of the employed topo-bathymetric laser sensor of ca. $1.6 \mathrm{~ns}$ results in a range discrimination distance of $24 \mathrm{~cm}$,
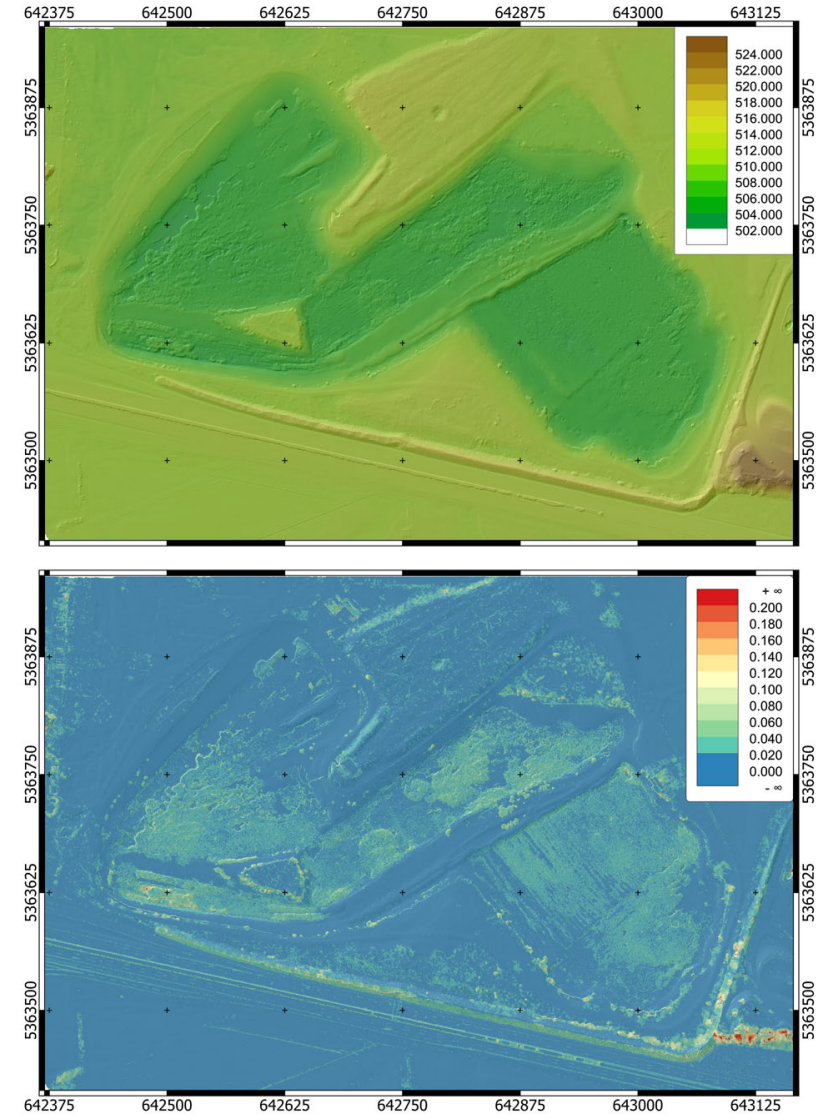

Figure 6. DTM of Lake Autobahnsee containing bare land surface and submerged topography; top: color coded elevation map superimposed with shaded relief map; bottom: color coded height precision map $\left(\sigma_{z}\right)$

thus, enabling multi-target detection for plants with a height less than $0.5 \mathrm{~m}$. In addition to the laser data, the concurrently acquired Coastal Blue and RGB image data are of further use for identification and characterization of the under-water vegetation (cf. Figure 9 below).

As described in Section 3.2, the RGB and Coastal Blue images were oriented independently using Pix4D mapper based on the 16-bit TIFF (Tiled Image File Format) imagery exported from the raw camera images using the CaptureOne software. For image matching, the multispectral RGB images need to be converted to single-banded pan-chromatic images. In the conversion process normally the green band gets the highest weight $(60-70 \%$, followed by red (20-30\%), and blue ( $10 \%)$. This preprocessing step, however, is not necessary for the monochromatic (singleband) Coastal Blue images. Figure 7 and Figure 8 show a perspective view of the RGB and Coastal Blue image bundles of Lake Autobahnsee containing images of 4 flight strips. The plots contain the image tie points, the control points (blue circles), the camera positions, and the bundle of image rays intersecting in control point 25 .

It is noted here that matches should generally be avoided in the wetted perimeter due to the bending of the image rays at the airwater interface. Thus the collinearity equations, constituting the the underlying mathematical model in both photogrammetry and Structure from Motion (SfM), are violated in these areas and a more complex model including the water surface as an additional unknown would be needed (Mulsow, 2010). Figure 7 shows that 

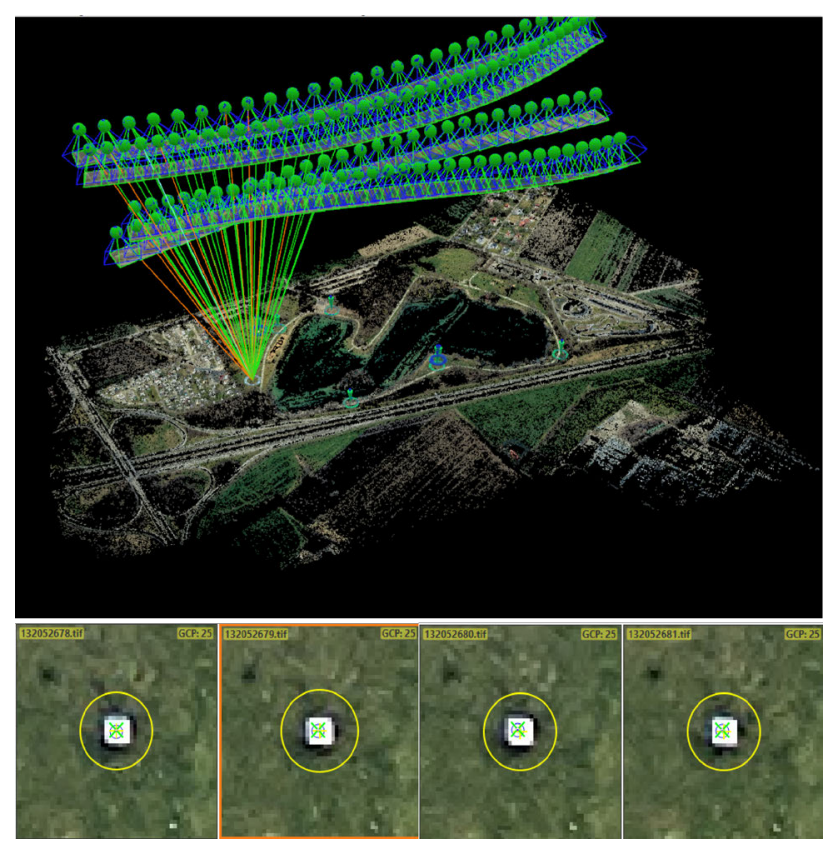

Figure 7. RGB image bundle block; top: 3D-view of automatically matched image tie points, image rays intersecting at manually measured control point 25 ; bottom: control point 25 displayed in images 52678-81
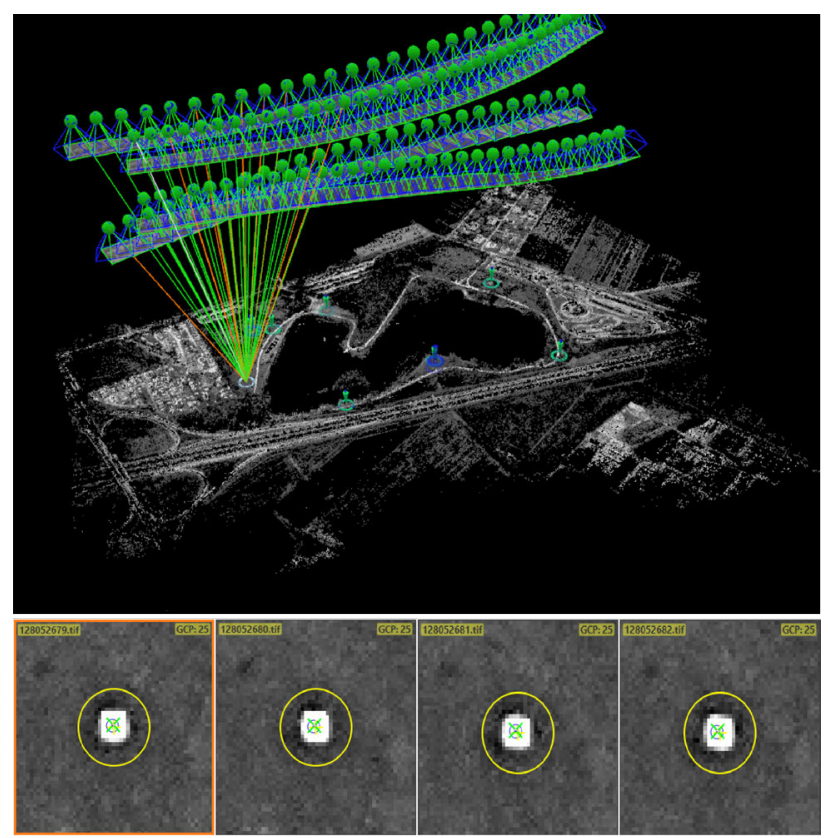

Figure 8. Coastal blue image bundle block; top: 3D-view of automatically matched image tie points, image rays intersecting at manually measured control point 25 ; bottom: control point 25 displayed in images 52679-82

the tie point density within the lake is much lower compared to the surrounding dry land area. The main reasons for the reduced tie point density in water are (i) blurring of the bottom features due to scattering in the water column and (ii) rejection of potential matches due to irregular displacements of the lake bottom feature points resulting from image ray refraction at the slightly wavy water surface. Still it can be observed from Figure 7 that several lake bottom tie points exist, as (i) the water surface was very calm during data capturing, (ii) the depth of Lake Autobahnsee
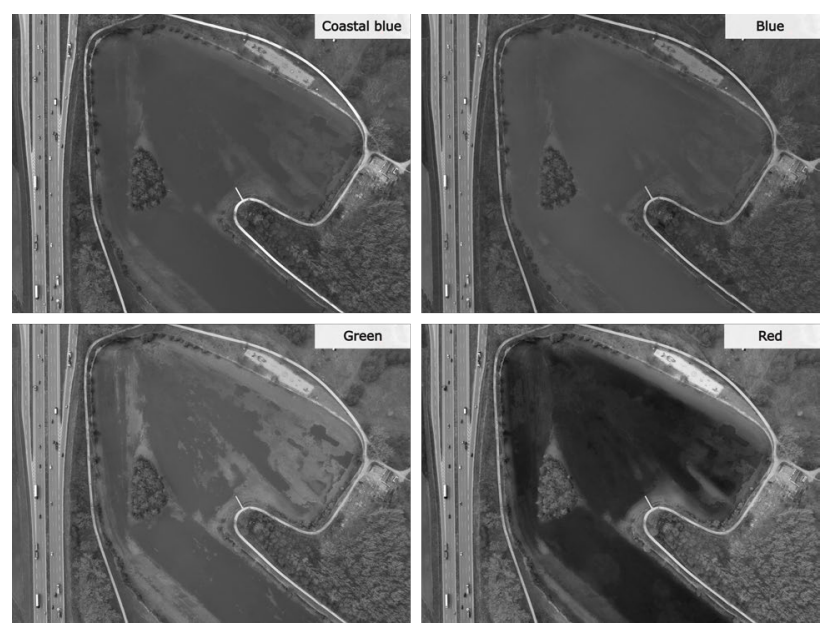

Figure 9. Color channels of aerial image 52922

\begin{tabular}{|c|c|c|c|}
\hline & unit & RGB & Coastal Blue \\
\hline \# tie point observations & - & 2495546 & 1856068 \\
\hline \# 3D tie points & - & 566680 & 520697 \\
\hline \# matched tie points/image & - & 19894 & 15392 \\
\hline mean reprojection error & [pix] & 0.117 & 0.140 \\
\hline ME (X) of control points & [m] & 0.006 & 0.003 \\
\hline ME (Y) of control points & {$[\mathrm{m}]$} & 0.004 & 0.012 \\
\hline $\mathrm{ME}(\mathrm{Z})$ of control points & {$[\mathrm{m}]$} & -0.009 & 0.018 \\
\hline RMSE (X) of control points & [m] & 0.026 & 0.024 \\
\hline RMSE (Y) of control points & [m] & 0.035 & 0.030 \\
\hline RMSE (Z) of control points & [m] & 0.054 & 0.065 \\
\hline
\end{tabular}

Table 2. Results of bundle block adjustment

is limited (max. depth: $4.6 \mathrm{~m}$, mean depth: $2.1 \mathrm{~m}$ ), and (iii) the intersection angles of image rays from consecutive exposures are small (ca. $4^{\circ}$ ) limiting the (relative) pixel displacement due to refraction. Interestingly, there are much fewer water bottom tie point in the Coastal Blue image block. While this is desirable for image orientation (given enough tie points in the surrounding dry area), it is a first indication that image texture is generally lower compared to the pan-chromatic images derived from RGB. This topic is discussed in more detail below.

Representative bundle block adjustment results are listed in Table 2. The RGB-based variant hereby outperforms Coastal Blue

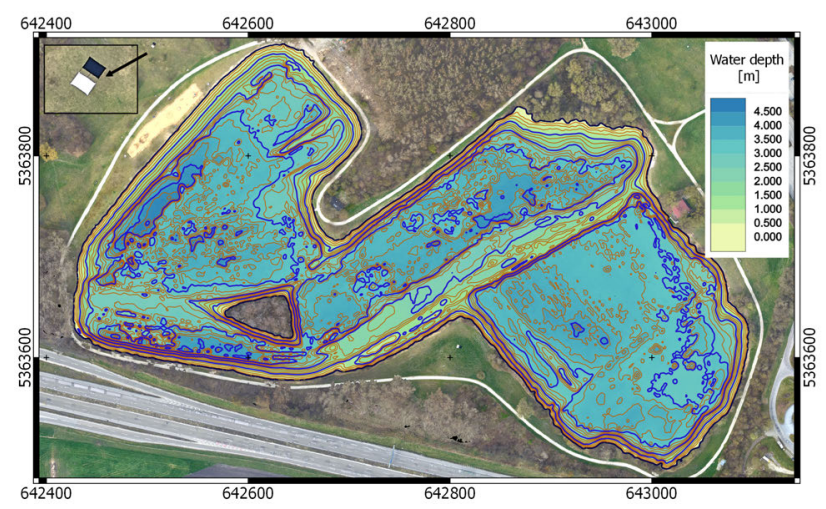

Figure 10. Digital orthophoto map derived from DigiCAM 100 RGB images superimposed with LiDAR derived water depth map (color coded) and depth contours (thin brown lines: $25 \mathrm{~cm}$ contours, thick blue lines: $1 \mathrm{~m}$ contours). Detail in upper left corner: radiometric control patch 

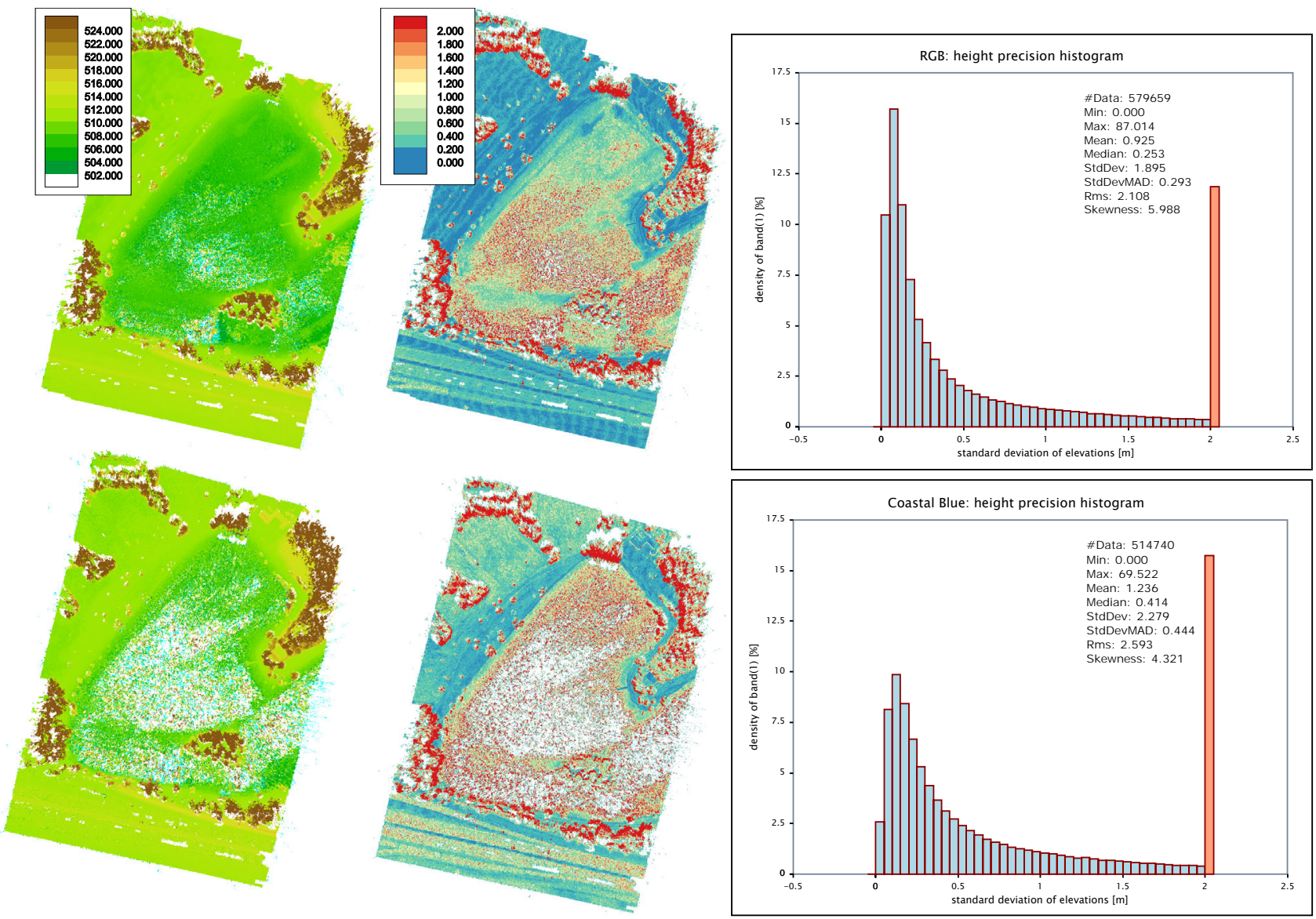

Figure 11. Results of Through-Water Dense Image Matching for stereo pair 52920-52921 based on RGB (top) and Coastal Blue

(bottom) images; left: Color coded map of elevations [m]; middle: height precision [m]; right: height precision histogram

in all tested categories relying on automated processing (i.e. feature detection, tie point matching). In contrast to that, the absolute discrepancies at the manually measured control points do not favor the one or the other image basis. This basically means that, in general, the RGB images and the pan-chromatic channel derived thereof exhibit better image texture (needed for automatic feature point extraction and tie point matching), but the Coastal Blue images are equally interpretable for the human operator, especially in the case of well defined checker board targets.

Visual inspection of Figure 9, showing the separate color bands of a concurrent RGB/Coastal Blue exposure, further supports the above quantitative assessment. The figure clearly features the superiority of the green channel with respect to the visibility of bottom texture. The Coastal Blue channel performs better than the blue band concerning the visibility of submerged bottom features and the red channel, in turn, is most affected by the attenuation within the water column, with deeper areas appearing darker. For the image orientation, however, the dry land part is most influential, as most of the tie points were identified there. Whereas the lane marks of the motorway clearly stand out from surrounding asphalt in the Coastal Blue band, vegetated areas appears clearest in the red channel due to the better reflectivity of green vegetation in the red domain of the electromagnetic spectrum.

In water, the gray values of the red channel are obviously correlated with the depth of this shallow lake. While this is beneficial for spectrally based depth estimation (Lyzenga et al., 2006; Legleiter et al., 2009), the availability of bottom texture is most important for deriving bathymetry via multi-media photogram- metry (Maas, 2015) in general and through-water dense image matching in particular (Mandlburger, 2018).

Figure 10 shows a composite map of Autobahnsee consisting of the digital orthophoto obtained from the RGB images of 3 flight lines superimposed with the LiDAR derived water depth map (color coded) and the $0.25 \mathrm{~m}$ (brown) and $1 \mathrm{~m}$ (blue) depth contours, respectively. The LiDAR sensor delivered gapless depth measurements for the entire lake (mean/max. water depth ca. 2.1/4.6 m). Area wide depth estimates were also obtained via through-water dense matching based on the RGB and Coastal Blue images using Pix4D and SURE (Rothermel et al., 2012; Wenzel et al., 2013). While the LiDAR sensor provided consistent depth estimates especially in the barren gravel areas, the photogrammetrically derived point clouds expectedly produced poorer results w.r.t. height precision.

For a single stereo image pair (52920-52921) Figure 11 shows the resulting DEM derived as the median of all successfully matched $3 \mathrm{D}$ points in cells of $50 \mathrm{~cm}$ size. In the top row the results obtained from the RGB stereo pair are displayed as a color coded elevation map (left), a color coded height precision map (middle), and a height precision histogram (right). The same products in the bottom row show the respective results for the Coastal Blue based DEM. While the RGB-derived DEM only contains sporadic void pixels (white), much more data voids are observed in the Coastal Blue based DEM. The RGB DEM outperforms the Coastal Blue DEM not only in completeness but also in height precision, indicated by the higher amount of red pixels in the height precision maps. The superiority can also be seen from the histograms 

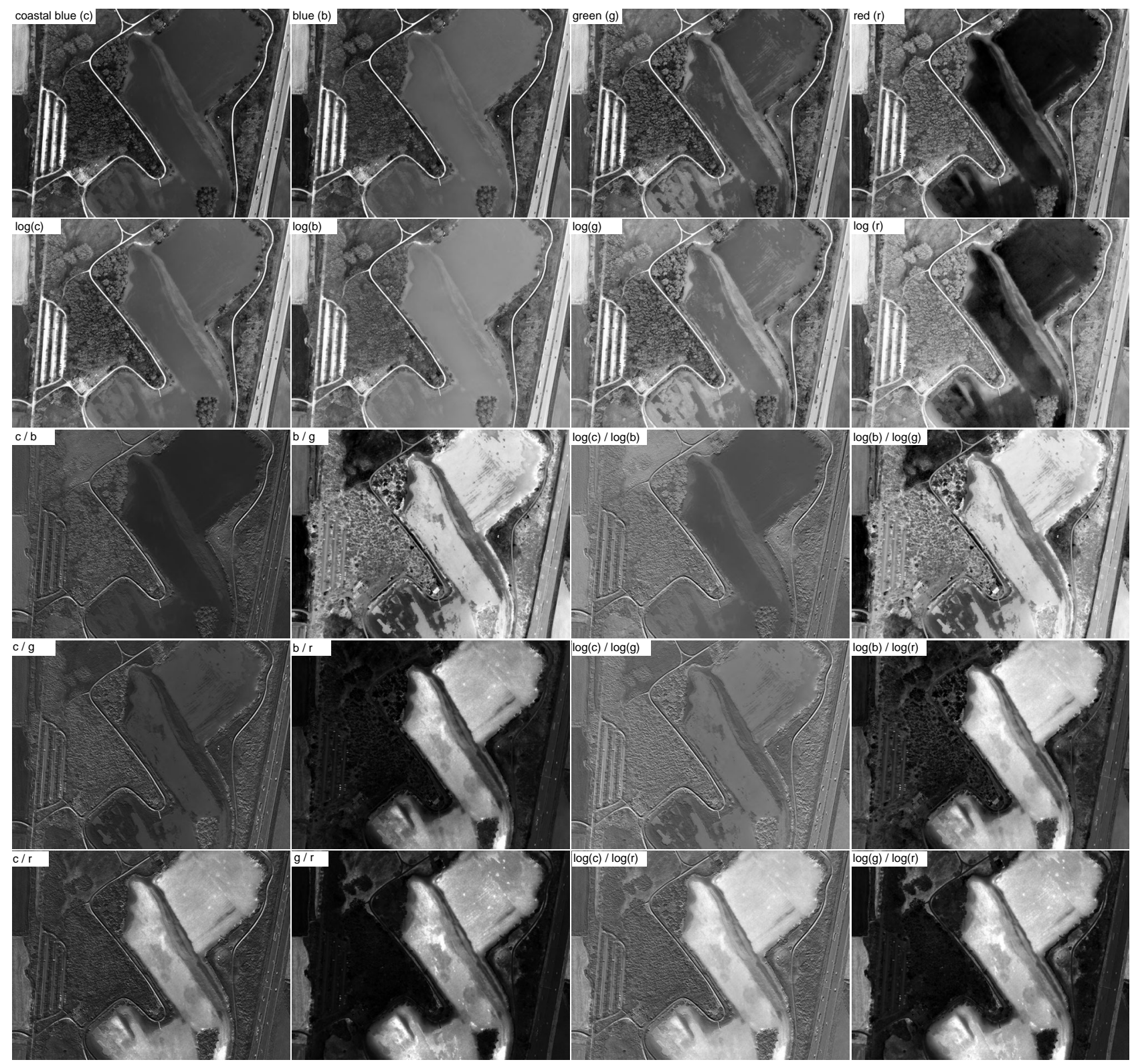

Figure 12. Image features derived from the four color channels; top row: coastal blue, blue, green and red band; second row: (natural) logarithms of color bands; lower left block: simple ratios of color bands; lower right block: ratios of logarithms of color bands

showing a higher peak in the $0-20 \mathrm{~cm}$ precision range compared to the Coastal Blue results. Also the key statistics underline the better performance of RGB (e.g. median: RGB=0.25 vs Coastal Blue $=0.41 \mathrm{~m})$.

This clearly shows that the Coastal Blue band does provide bathymetric capabilities, not only in the coastal domain (Miecznik and Grabowska, 2012) but also for inland water bodies. But it is also evident that especially the green band performs better from a photogrammetric point of view. However, next to the assessment of the applicability of Coastal Blue in the context of multi-media photogrammetry, another question of interest was, if this wavelength provides added value in combination with other bands. So far, this question was addressed empirically by assessing different feature images calculated either from the raw bands, their (natural) logarithms, band ratios, and ratios of the logarithm of the respective bands. Figure 12 show an exemplary set of tested features for an image of the Autobahnsee dataset. From visual inspection, the following conclusions can be drawn when con- centrating on the water domain:

- Compared to the raw bands (top row of Figure 12), the logarithm of the bands ( $2^{n} d$ row) appear both brighter and more textured.

- The ratio of the band logarithms are brighter than the respective ratios of the raw bands. However, this does not necessarily hold for the contrast (which is the main parameter for multi-media photogrammetry).

- Any ratio with the red band in the denominator produces a bright image of the submerged areas as red is most affected by signal absorption and thus dark, especially in deep water areas. Due to the fact that all other bands are also subject to signal attenuation within the water column, the ratio stays in reasonable ranges. In other words, the gain in brightness does not come with a loss of contrast.

- From all features with Coastal Blue involvement, the raw band ratio with the red band (i.e. $c / r$, lower left image in Figure 12) delivers the highest contrast. However, the green- 
to-red ratio provides more contrast based on a purely visual assessment.

- No single feature image can be named as best (in terms of delivering the highest contrast). Instead, some bottom features are best represented in the one feature image while other areas deliver a better contrast in a different feature image. This opens the potential future research topic of finding the optimal feature composite for deriving bathymetry either via multi-media photogrammetry or via spectrally based depth estimation (i.e. establishing a color-to-depth relation).

By the time of writing of writing this manuscript, a respective quantitative evaluation is work in progress.

\section{CONCLUSIONS AND OUTLOOK}

In this contribution we reported on an experimental airborne data acquisition with a topo-bathymetric laser scanner (Riegl VQ-880G) and two IGI DigiCAM 100 cameras. The experiment was planned to investigate the benefit of using Coastal Blue $(\lambda=400$ $460 \mathrm{~nm}$ ) for mapping bathymetry and detecting and characterizing submerged features (e.g. different types of under-water vegetation). While data processing and analysis is still in progress, first results indicate that, in general, the green band is more useful for multi-media photogrammetry purposes as signal attenuation within the water column is low and bottom features (e.g. vegetation) stand out well from the surrounding underground (e.g. gravel). For image orientation and through-water dense image matching, better precision could be achieved based on the RGB images compared to Coastal Blue.

So far, we can state that the Coastal Blue band adds an extra water penetrating channel, thus increasing the potential combinations, e.g., for identifying optimal band ratios for spectrally derived depth estimation (Legleiter et al., 2009; Legleiter, 2016) or for deriving a composite feature image optimizing the local contrast (i.e. texture) for multi-media photogrammetry (Maas, 2015; Mandlburger, 2018). In any case, the topo-bathymetric LiDAR data has proven its worth as a reliable reference source for validating the depth estimates from passive optical remote sensing. Currently quantitative investigations are work in progress and will allow to draw more reliable conclusions concerning the added value of using high-resolution Coastal Blue imagery for bathymetric mapping.

\section{ACKNOWLEDGEMENTS}

The presented investigation was conducted within the project "Bathymetry by Fusion of Airborne Laser Scanning and MultiSpectral Aerial Imagery"' funded by the German Research Foundation (DFG). The authors want to thank Christian Mayr and Hannes Nuebel (undergraduate students at the University of Stuttgart) for helping with data processing.

\section{References}

Anderson, N. T. and Marchisio, G. B., 2012. WorldView-2 and the evolution of the DigitalGlobe remote sensing satellite constellation: introductory paper for the special session on WorldView-2. In: Proc.SPIE, Vol. 8390, pp. 8390-8390-15.

Glira, P., Pfeifer, N. and Mandlburger, G., 2016. Rigorous strip adjustment of UAV-based laserscanning data including timedependent correction of trajectory errors. Photogrammetric Engineering \& Remote Sensing 82(12), pp. 945-954.
Guenther, G. C., Cunningham, A. G., Laroque, P. E. and Reid, D. J., 2000. Meeting the accuracy challenge in airborne lidar bathymetry. In: Proceedings of the 20th EARSeL Symposium: Workshop on Lidar Remote Sensing of Land and Sea, Dresden, Germany.

Kraus, K. and Pfeifer, N., 1998. Determination of terrain models in wooded areas with airborne laser scanner data. ISPRS Journal of Photogrammetry and Remote Sensing 53, pp. 193-203.

Legleiter, C. J., 2016. Inferring river bathymetry via Image to Depth Quantile Transformation (IDQT). Water Resources Research 52(5), pp. 3722-3741.

Legleiter, C. J., Dar, A. R. and Rick, L. L., 2009. Spectrally based remote sensing of river bathymetry. Earth Surface Processes and Landforms 34(8), pp. 1039-1059.

Lyzenga, D. R., Malinas, N. P. and Tanis, F. J., 2006. Multispectral bathymetry using a simple physically based algorithm. IEEE Transactions on Geoscience and Remote Sensing 44(8), pp. 2251-2259.

Maas, H.-G., 2015. On the Accuracy Potential in Underwater/Multimedia Photogrammetry. Sensors 15(8), pp. 1814018152.

Mandlburger, G., 2018. A Case Study on Through-Water Dense Image Matching. In: ISPRS TC II Mid-term Symposium Towards Photogrammetry 2020, Vol. XLII-2, pp. 659-666.

Mandlburger, G., Pfennigbauer, M. and Pfeifer, N., 2013. Analyzing near water surface penetration in laser bathymetry A case study at the River Pielach. In: ISPRS Annals of the Photogrammetry, Remote Sensing and Spatial Information Sciences, Vol. II-5/W2, pp. 175-180.

Miecznik, G. and Grabowska, D., 2012. Worldview-2 bathymetric capabilities. In: Proc.SPIE, Vol. 8390, pp. $8390-8390-$ 10.

Mulsow, C., 2010. A flexible multi-media bundle approach. In: ISPRS Comission V Symposium, Newcastle upon Tyne, UK, Vol. XXXVIII-5, pp. 472-477.

Pfeifer, N., Mandlburger, G., Otepka, J. and Karel, W., 2014. OPALS - A framework for Airborne Laser Scanning data analysis. Computers, Environment and Urban Systems 45, pp. 125 -136 .

Ressl, C., Kager, H. and Mandlburger, G., 2008. Quality Checking Of ALS Projects Using Statistics Of Strip Differences. In: International Archives of the Photogrammetry, Remote Sensing and Spatial Information Sciences 37 (Part B3b), pp. 253 260 .

Rothermel, M., Wenzel, K., Fritsch, D. and Haala, N., 2012. SURE: Photogrammetric surface reconstruction from imagery. In: Proceedings of the Low Cost $3 D$ Workshop, Berlin.

Shannon, C. E., 2001. A mathematical theory of communication. SIGMOBILE Mob. Comput. Commun. Rev. 5(1), pp. 3-55.

Wenzel, K., Rothermel, M., Haala, N. and Fritsch, D., 2013. SURE the ifp Software for Dense Image Matching. In: D. Fritsch (ed.), Photogrammetric Week '13, Wichmann/VDE Verlag, Berlin \& Offenbach, pp. 59-70. 